\title{
Fluorescence dynamics of an ensemble of cold atoms
}

\author{
Sergey Bozhokin*, and Alexey Kuraptsev \\ Peter the Great St. Petersburg Polytechnic University, 195251 St. Petersburg, Russia
}

\begin{abstract}
We have calculated the fluorescence dynamics of cold atoms excited by pulsed radiation. We have considered both Radon-Nikodym spectral method and multi-stage relaxation model describing collective effects in cold atomic gases. Relaxation times and amplitudes both at short times (superradiance) and long times (subradiance) have seen obtained.
\end{abstract}

The dynamics of the afterglow of atomic ensembles cooled to ultra-low temperatures is important in view of the diagnostics of such objects [1-4]. The purpose of this work is a theoretical analysis of the relaxation of fluorescence both in strong external electric field and in the absence of external field. Upon the solution of Schrodinger equation, we take into account the interaction of atoms with external electromagnetic field, interatomic resonant dipole-dipole interaction and spontaneous decay of the atomic excited states.

The specific calculations have been performed for the cold atomic cloud of spherical shape with radius $R=10 \lambda$, atomic density $n_{0} \lambda^{3}=0.2$, where $\lambda=1 / k, k$ is the wave number of probe radiation. The duration of excitation pulse is $\tau_{L} / \tau_{a}=0.1$, where $\tau_{a}$ is the lifetime of the excited state. In order to analyze the fluorescence, we introduce the function $Y(x)=\ln \left(I^{2}(t) /\left(I_{\max } I_{\min }\right)\right) / \ln \left(I_{\max } / I_{\min }\right)+x$. In this equation, the time $t$ changes in a range $t=[0, T], T$ is the time of observation, $x=2 t / T-1$ is the dimensionless time, which changes in a range $-1 \leq x \leq 1, I(t)$ is the intensity of fluorescence, $I(0)=I_{\max }$, $I(T)=I_{\min }$. From the definition, one can see that the function $Y(x)$ changes in a range $-1 \leq Y(x) \leq 1$. Orthogonal real Chebyshev polynomials $\left\{f_{n}(x)\right\}$ allow one to calculate the elements of the symmetric matrix of the signal $Y(x)$.

Using the Radon-Nikodym method, we have built the histograms of the distribution of the eigenvalues of such a signal matrix. The discrepancy between the relaxation curves $Y(x)$ manifests itself in the different diapasons of the eigenvalues of signal matrices. Skewness parameter of these histograms allows one to distinguish the fluorescence kinetics of cold atoms in a strong electric field $(E \neq 0)$ and in the absence of external field $(E=0)$.

\footnotetext{
* Corresponding author: bsvjob@mail.ru
} 
We have also considered multi-exponential relaxation model $I(t)=\sum_{i=1}^{N} B_{i} \exp \left(-t / \tau_{i}\right)$ with constant amplitudes $B_{i}$ and times $\tau_{i}$. In the case $N=4$, the root-mean-square deviation $\sigma$ between calculated relaxation curves and the results of multi-exponential model is $\sigma$ $=0.01$. Typical values of the amplitudes and relaxation times both at short times (superradiance) and long times (subradiance) have seen obtained. At the initial stage of evolution, relaxation time of the superradiance in the presence of electric field $\tau_{1}(E)$ is larger than that without external field, $\tau_{1}(E)<\tau_{1}(0)$. In case of superradiance, multi-stage processes described by the multi-exponential model display the mechanisms of fluorescence relaxation of the whole cluster of cold atoms at different time intervals.

The developed technique of the analysis of the afterglow dynamics upon the excitation by pulsed radiation gives one the opportunity to control the parameters of cold atomic ensembles and impurity centers embedded in a transparent dielectric experimentally. Proposed analysis of the fluorescence dynamics allows one to find out various collective effects caused by the photon exchange between atoms in dense and cold atomic clouds. Furthermore, this method allows one to obtain the lifetimes of different collective states that determine the decay rate of fluorescence at different time intervals after the end of excitation pulse.

This work was supported by the Russian Science Foundation (Grant No. 17-12-01085).

\section{References}

1. J.R. Lakowicz, Principles of Fluorescence Spectroscopy. Third Edition. (NY, Springer, 2006).

2. L.V. Hau, Nature Photonics, 2, 451 (2008).

3. A.S. Kuraptsev, I.M. Sokolov, M.D. Havey, Phys. Rev. A, 96, 023830 (2017).

4. S.V. Bozhokin, I.M. Sokolov, Opt. Spectrosc., 125(3), 317 (2018).

5. A. Bobyl, A. Zabrodskii, M. Kompan, V. Malyshkin, O. Novikova, E. Terukov, D. Agafonov, arxiv:1611.07386. (2018). 